\title{
HIV -Associated benign lymphoepithelial parotid cysts: a rare case report
}

\author{
Brihaspati Sigdel $^{* 1}$ (iD), Priti Acharya ${ }^{2}$, Rajesh Maharjann', Kusheswar Sah¹ \\ 'Department of Otolaryngology \& Head and Neck Surgery, Gandaki Medical College, ${ }^{2}$ Regional program Coordinator, AIDS \\ Healthcare Foundation, Pokhara Academy Health Sciences, Pokhara, Nepal
}

\begin{abstract}
Approximately one to $10 \%$ of patients with Human Immunodeficiency virus (HIV) infection associated with salivary gland disease. The presence of Benign lymphoepithelial cyst (BLEC) in the parotid gland is an indicator of HIV Infection. The diagnosis is usually based on a clinical course and HIV confirmatory blood testing. We have reported a case of a 35 years old Nepalese male patient with swelling of the bilateral parotid glands and HIV associated BLEC confirmed by Rapid diagnostic test kit (Determine unigold stat pack)
\end{abstract}

Key words: Benign Lymphoepithelial Cyst, HIV positive, rapid diagnostic test kit

\section{*Correspondence:}

Dr. Brihaspati Sigdel

Associate Professor \& Head of Department Department of Otolaryngology \& Head and Neck Surgery

Gandaki Medical College Teaching Hospital, Pokhara, Nepal.

Email: brihassig1@gmail.com

Phone No: +977-9856030090

Submitted: August 30, 2020

Accepted: June 24, 2021

To cite: Sigdel B, Acharya P, Maharjhan R, Sah K. HIV-Associated benign lymphoepithelial parotid cysts: a rare case report. JGMC Nepal. 2021;14(1):73-4.

DOI: $10.3126 /$ jgmcn.v14i1.30938

\section{INTRODUCTION}

Parotid swelling in HIV patients is associated with Benign lymphoepithelial cyst. ${ }^{1}$ BLEC is characterized by bilateral parotid gland swelling and cervical lymphadenopathy. ${ }^{2}$ The incidence of BLEC has increased with the emergence of HIV and its prevalence decrease with the treatment of antiretroviral therapy. The diagnosis of HIV associated with BLEC was made through clinical examination, ultrasonography, and rapid diagnostic test. The reported case of 35 years old male patient with parotid benign lymphoepithelial cyst was found to be HIV positive.

\section{CASE DESCRIPTION}

A 35 years male presented to the ENT out patient department at Gandaki Medical College, Pokhara, Nepal in August 2019 with enlarging mass at the bilateral parotid region for six months. It was painless and not associated with fever. On examination, the patient had swelling bilateral parotid region around $5 \times 4 \mathrm{~cm}$ size and normal overlying skin., no discharge, or pulsation. The swelling was nontender with well-defined margin, smooth boundary, cystic and overlying skin was free and mobile. The facial nerve function was intact. He had multiple mobile lymph nodes at level II, III and V and tender bilateral axillary lymphadenopathy. Intraoral examination was within the normal limit with free flow of saliva from the Stensen's duct. Ultrasound of the parotid showed multiple thin wall cysts with large one measuring $2.5 \times 2.0 \mathrm{~cm}$. (Fig.1) Fine needle aspiration cytology (FNAC) finding revealed scattered foamy macrophages and anucleated squamous cell in a proteinaceous background. (Fig.2) HIV was confirmed by blood test with a Determine, Uni-Gold STATPACK. 


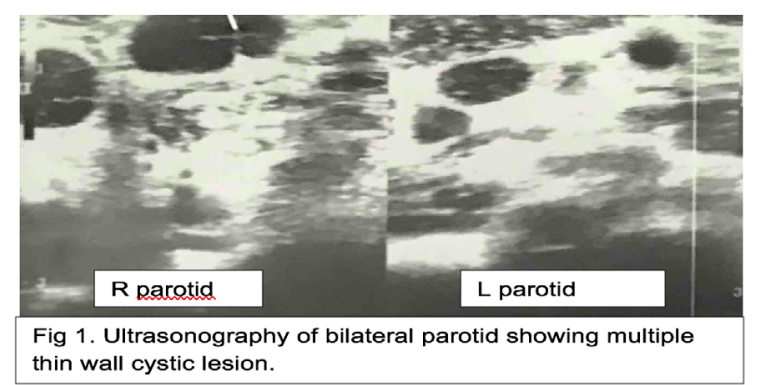

Figure 1. Ultrasonography of bilateral parotid showing multiple thin wall cystic lesions.

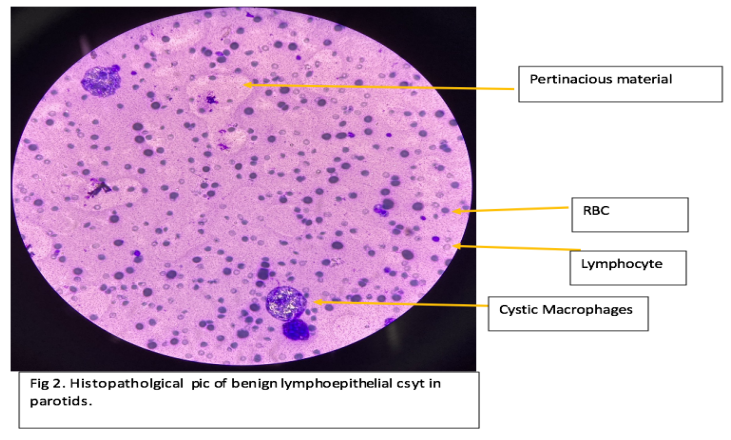

Figure 2: Histopathological picture of benign lymphoepithelial cyst in parotid

\section{DISCUSSION}

BLEC is a cystic disease of the parotid gland which has a differential diagnosis of warthin's tumor and cystic degeneration of benign and malignant tumors. ${ }^{1}$ The proliferation of BLEC has been shown to increase with HIV replication. The exact pathology of this disease remains unknown. ${ }^{4}$ The supportive hypothesis is that HIV related reactive lymph node proliferation occurs in the intra parotid lymph node or HIV infected cell can migrate in the parotid gland that induces ductal obstruction and cyst formation. It is diagnosed on the basis of medical history, clinical examination, parotid ultrasound imaging and blood test with a Determine, Uni-Gold STAT-PACK.

Ultrasound detects multiple thin-wall cysts and diffuse cervical lymphadenopathy. FNAC can exclude malignancy. Malignancy occurs in less than $1 \%$ of HIV associated with cystic lesions. FNAC is a safe investigation that shows groups of lymphoid cells include tangible body macrophage, dendritic cell, histiocytes, and multiple epithelial cells. ${ }^{5}$ The management option for BLEC remains controversial. It includes aspiration, ART, sclerotherapy, radiation therapy, and surgery. We had given antiretroviral therapy including Tenofovir, Lamivudine, and Efavirenz in combination and parotid swelling was decreasing in size in response to treatment. ${ }^{1,4}$ We did superficial conservative parotidectomy in HIV negative patients. However, some reports do not recommend surgery as a first-line treatment in HIV positive patients. It is a good option for those patients who had poor responses to antiretroviral therapy.

\section{CONCLUSION}

Lymphoepithelial lesions can manifest as cystic parotid swelling in HIV patients. Hence, they should be considered as a differential diagnosis in benign parotid cystic swelling. HIV associated BLEC confirmed by the Rapid Diagnostic test kit and the Conservative management is the preferred approach for the treatment of benign lymphoepithelial lesions.

\section{Conflict of Interest: None}

\section{REFERENCES}

1. Dave SP, Pernas FG, Roy S. The benign lymphoepithelial cyst and a classification system for lymphocytic parotid gland enlargement in the pediatric HIV population. The Laryngoscope. 2007 Jan;117(1):106-13. DOI: 10.1097/01. mlg.0000246196.35413.35

2. Amatya PR, Limbu TR, Baral SJ, Bastakoti S. Lymphoepithelial cyst of the parotid in HIV negative individuals: a case report. Journal of Chitwan Medical College. 2019; 9: 81-2. DOI: $10.3126 /$ jcmc.v9i4.26910

3. Mourad WF, Patel S, Young R, Khorsandi AS, Concert C, Shourbaji RA, etal. Managementalgorithm for HIV-associated parotid lymphoepithelial cysts. European Archives of Oto-Rhino-Laryngology. 2016 Oct;273(10):3355-62. DOI: 10.1007/s00405-016-3926-4

4. Sekikawa Y, Hongo I. HIV-associated benign lymphoepithelial cysts of the parotid glands confirmed by HIV-1 p24 antigen immunostaining. Case Reports 2017; 2017: bcr-2017221869.DOI: 10.1136/bcr-2017-221869

5. Shaha AR, DiMaio T, Webber C, Thelmo W, Jaffe BM. Benign lymphoepithelial lesions of the parotid. The American journal of surgery. 1993 Oct 1;166(4):403-6. DOI: 10.1016/ S0002-9610(05)80342-3 